\title{
A Research on Deep Learning Advance for Landslide Classification using Convolutional Neural Networks
}

\author{
Jaishankar Bhatt, Akash Gangwar, Rahul Nijhawan, Durgaprasad Gangodkar
}

\begin{abstract}
Landslides can easily be tragic to human life and property. Increase in the rate of human settlement in the mountains has resulted in safety concerns. Landslides have caused economic loss between 1-2\% of the GDP in many developing countries. In this study, we discuss a deep learning approach to detect landslides. Convolutional Neural Networks are used for feature extraction for our proposed model. As there was no source of an exact and precise data set for feature extraction, therefore, a new data set was built for testing the model. We have tested and compared this work with our proposed model and with other machine-learning algorithms such as Logistic Regression, Random Forest, AdaBoost, K-Nearest Neighbors and Support Vector Machine. Our proposed deep learning model produces a classification accuracy of $96.90 \%$ outperforming the classical machine-learning algorithms.
\end{abstract}

Keywords-Convolutional Neural Network, Deep Learning, ReLu, Pooling.

\section{INTRODUCTION}

A landslide is nothing but a motion of debris, large rocks or terrain down a slope due to gravity [1]. Landslides happen in a variety of environment portrayed by either gentle or steep slope inclinations ranging from mountains to coastal reefs and also even underwater. The factors such as an earthquake, a heavy rainfall, floods, construction work on the slope which triggers the landslides by affecting the stability of the slope.

Various factors such as the higher rate of unplanned urbanization, deforestation, and unpredicted rainfall resulting in the increase of landslide problems which seems to be more challenging in the future. On July 30, 2014, 151 people died and hundreds were missing in the Malin village in Maharashtra due to landslide [2]. In July 2013 more than 5700 people were dead due to landslides caused by Kedarnath floods in Uttarakhand [3]. In the future, the number of landslides will increase due to the effects of excessive rainfall and changing climatic conditions. In this manner, the landslide has turned out to be one of the hottest subjects for researchers. The precise estimation of the landslide is still a challenging real-world problem.

Landslides are hazards for human life and economy of a country, therefore, to minimize the damages and dangers to the people and their property many efforts have been made in the past to know the spatial and temporal distribution of landslides and to understand how to control them [4].

Revised Manuscript Received on April 12, 2019. India.

Jaishankar Bhatt, Dept. of CSE, GEU, Dehradun (Uttarakhand), Dr. India.

Dr. Durgaprasad Gangodkar, Dept. of CSE, GEU, Dehradun (Uttarakhand), India.
Akash Gangwar, Dept.of CSE, GEU, Dehradun (Uttarakhand), India.

Landslide classification is a new approach for detecting landslides. Landslide detection study will help in detecting the landslides and help early warning signs so that immediate safety actions should be taken.

In recent times, deep learning has brought advances in processing images, video, audio, and speech. It permits computational models that comprise multiprocessing layers to learn with representation of data with multi-level abstraction. Deep learning models find patterns in data sets by utilizing backpropagation to specify how a machine ought to alter its weights that are utilized to process the representation of data in each layer from the past layer [5]. This paper discusses the deep learning framework for landslide classification which will be helpful in classifying and detecting landslide areas. We have proposed the Convolution Neural Network (CNN) model approach for landslide classification. We observed that the CNN approach outperformed the traditional machine learning methodologies such as KNN, RF, SVM, Logistic Regression and AdaBoost. The unavailability of the data set was a challenge faced by our model. We needed effective and precise resources of feature extraction so we created our own high-resolution image data set of landslide-prone areas.

\section{LITERATURE REVIEW}

In the past years, many researchers have shown their immense interest in the area of landslide. Landslide classification using the deep neural network is a newly pursued subject in which landslide is detected in the images which will be helpful in distinguishing landslide regions in maps and through images of sites prone to landslides.

Truong et al. [6] have used ensemble technique which is a fusion of Bagging Ensemble technique which is a fusion of Bagging Ensemble and Logistic Model Tree for refining the performance of landslide susceptibility model.

Oh and Lee [7] proposed data mining approaches such as Artificial Neural Network and Boosted Tree for landslide susceptibility modeling giving the validation result of $82.5 \%$ and $90.79 \%$ respectively. Ramesh [8] discusses the design and deployment of a landslide detection system using a Wireless Sensor Network system to detect landslides. To detect landslides of five different types Martha, Kerle, Western, Hetten, and Kumar [9] presents an intelligent classification approach with the thresholds of diagnostic parameters derived from if-means cluster analysis with an overall test accuracy of $77.7 \%$.

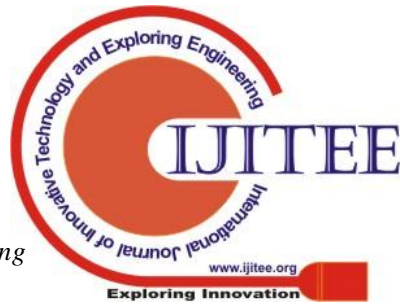




\section{DATA COLLECTION}

Data collection is a very important process to capture quality evidence that allows analysis to lead to the design of considerable and reliable solutions to the problem that has been put forward. In this study, due to the unavailability of open source image data set on landslides, we created an image data set for our proposed model. A binary class data set i.e. landslide and non-landslide were built for the problem. We collected good quality images (Fig. 1) from various sources like google images and different websites. A good quality data set of about 260 images was built for training and testing our proposed deep learning model. Models in machine learning need training data on which they are made learned the features and requires test data on which they are tested, so we divided the data set into training and testing parts. $80 \%$ of the data set is used as a training set in each category and the remaining $20 \%$ is used as a test set.

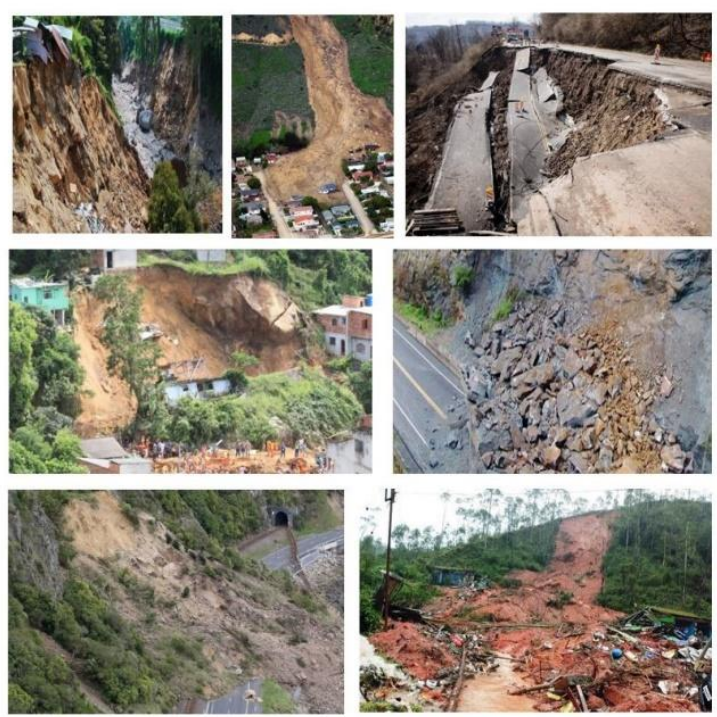

Fig. 1. Sample landslide images taken from our data set.

\section{METHODOLOGY}

We used deep learning framework i.e. CNN to extract significant features needed for detecting the landslide in our classes of data set i.e. landslide and non-landslide. We implemented our model on a machine consisting of $8^{\text {th }}$ generation Intel i5 processor with RAM of size $8 \mathrm{~GB}$, and a GPU (4GB Nvidia 940Mx).

The deep convolutional neural network processes the multi-array data, such as image data, audio, and video data. CNN constitutes basically three kinds of layers. The first layer is the convolutional layer in which the parameters comprise of a lot of kernels that can learn. The convolutional layer uses activation function, such as ReLu [10] i.e. Rectilinear Unit Function which produces nonlinearity in our model. The Second layer is pooling layer which diminishes the framework's computation by dimensionality reduction and furthermore controls overfitting. The used pooling is max-pooling which takes the greatest maximum value in each block. At last, the complex reasoning in the network is done in the third layer by taking all the neurons in the preceding layer and links it to each neuron it has.

We compared and contrasted our data set on the present state of the art algorithms (Logistic Regression, RF, AdaBoost, KNN, SVM) and proposed deep learning architecture on the basis of their performance.

\section{PROPOSED APPROACH}

A convolutional neural network architecture for the problem of landslide classification is presented in this paper. Our proposed architecture consists of 7 layers which are associated with weights, out of which there are 2 convolutional layers, 2 pooling layers, 1 flattening and 2 fully connected layers. In the Convolution stage, 32 activation maps are generated using $3 \times 3$ feature detector with input image size of $64 \times 64$ pixels and stride of 1-pixel. Convolutional layer uses the activation function called ReLu to increase the non-linearity in landslide images In Maxpooling, the pool size of $2 \times 2$ is taken for building the pooled feature map. After these two steps, we flatten our pooled feature map into a column-like long feature vector of an input image. It is passed through two fully connected layers which are formed from the multiple dense networks of neurons to predict whether the image is of a landslide or not. First, fully connected layer uses ReLu and other uses sigmoid activation functions to increase non-linearity. At the dense layer the feature vector is multiplied by the layer's weights, summed with biases and passed through an activation function for better results. Adam optimization algorithm [11] is used to update network weights iterative based on training data. This CNN architecture is then compiled to detect the images of class landslides or nonlandslides.

\section{RESULTS AND DISCUSSION}

The data set consisting of high-resolution photographs of landslides is very useful in building detailed feature maps. A total of 213 images for training and the remaining for test set were used. There was no overlap between the training and testing sets.

Our experimental algorithms obtained classification accuracy such as Logistic Regression (51.40\%), RF (80.70\%), AdaBoost (83.40\%), KNN (86.50\%), SVM $(92.70 \%)$. We noted that the classification accuracy of our suggested framework was the highest $(96.90 \%)$ with a sensitivity of $96.90 \%$ and precision of $96.90 \%$.

The new proposed deep learning CNN model performed very well with good accuracy. The role of $\mathrm{CNN}$ is to reduce the images into a form which is easier to process without losing features that are significant for getting better results. Table 1 provides an accuracy assessment of our work. 
Table 1. Accuracy Assessment

\begin{tabular}{|c|c|c|c|c|c|}
\hline Method & $\begin{array}{c}\text { Classification } \\
\text { Accuracy }\end{array}$ & Sensitivity & Precision & AUC & F1-Score \\
\hline Logistic Regression[12] & $51.40 \%$ & $51.40 \%$ & $26.40 \%$ & $50 \%$ & $34.8 \%$ \\
\hline RF[13] & $80.70 \%$ & $80.70 \%$ & $80.70 \%$ & $86.90 \%$ & $80.70 \%$ \\
\hline AdaBoost[14] & $83.40 \%$ & $83.40 \%$ & $83.40 \%$ & $83.30 \%$ & $83.40 \%$ \\
\hline KNN[15] & $86.50 \%$ & $86.50 \%$ & $88.60 \%$ & $93.50 \%$ & $86.20 \%$ \\
\hline SVM[16] & $92.70 \%$ & $92.70 \%$ & $93 \%$ & $92.20 \%$ & $92.70 \%$ \\
\hline Proposed Approach & $96.90 \%$ & $96.90 \%$ & $96.90 \%$ & $99.20 \%$ & $96.90 \%$ \\
\hline
\end{tabular}

We figured the ROC curve [17] for the assessment of the performance of our model (fig. 2) with other machine learning algorithms. Proposed Approach $(\mathrm{AUC}=99.20 \%)$ outperformed SVM (AUC=92.20\%), KNN (AUC $=93.50 \%), \quad$ AdaBoost $\quad(\mathrm{AUC}=83.30 \%), \quad \mathrm{RF}$ (AUC $=86.90 \%$ ) and Logistic Regression (AUC=50\%). The proposed deep learning CNN model has the highest predictive performance followed by SVM. It is because CNN is designed in a different way for processing dimensioned and ordered data. The convolution and maxpooling layer enforce weight sharing translationally. This demonstrates the mechanism of human visual cortex. It has shown to work very well for landslide classification.

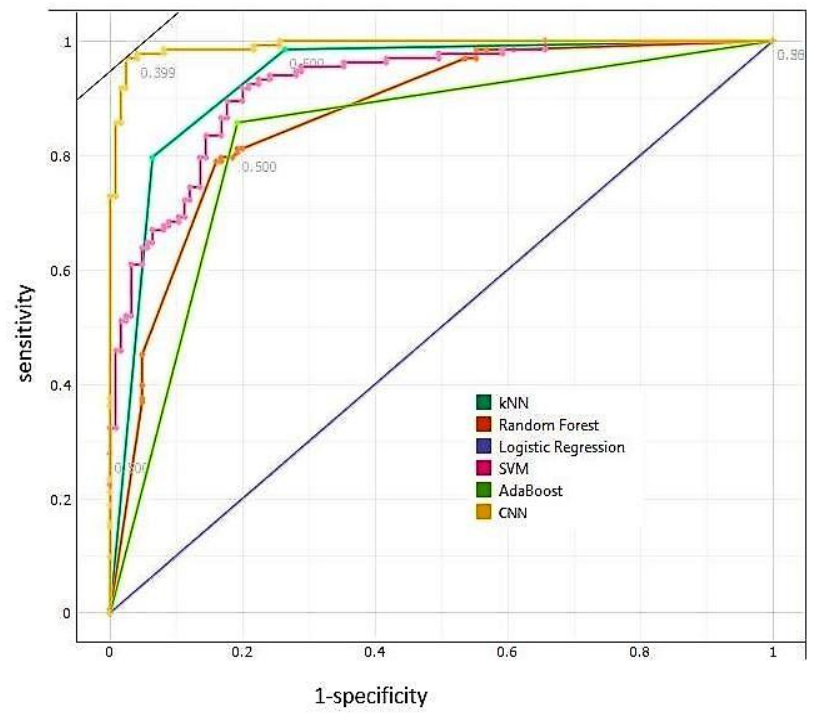

Fig. 2. ROC curve depicting various models and proposed approach

\section{CONCLUSION}

In our paper, we employed classical machine learning algorithms and a deep learning model to test their performance on our data set. We tested and compared their performance results and concluded that deep convolutional neural networks approach to detect landslides succeeded in getting an accuracy of $96.90 \%$. The proposed model is evaluated on various parameters such as ROC curve (AUC=99.20\%), Sensitivity (96.90\%), Precision (96.90\%) and F1-Score (96.90\%).

The purpose of this study may include early detection of the landslides so that rescue operations and safety measures can be taken rapidly to minimize the loss of life in landslide-hit areas. This work can help in making an early decision and land use planning in areas prone to landslides.

\section{REFERENCES}

1. Cruden, D.M., 1991. A simple definition of a landslide. Bulletin of Engineering Geology and the Environment, 43(1), pp.27-29.

2. Parkash, S., 2011. Historical records of socio-economically significant landslides in India. J South Asia Disaster Studies, 4(2), pp.177-204.

3. Jiménez-Perálvarez, J.D., El Hamdouni, R., Palenzuela, J.A., Irigaray, C. and Chacón, J., 2017. Landslide-hazard mapping through multi-technique activity assessment: an example from the Betic Cordillera (southern Spain). Landslides, 14(6), pp.1975-1991.

4. Varnes, D.J., 1984. Landslide hazard zonation: a review of principles and practice (No. 3).

5. Nijhawan, R., Verma, R., Bhushan, S., Dua, R. and Mittal, A., 2017, December. An Integrated Deep Learning Framework Approach for Nail Disease Identification. In 2017 13th International Conference on Signal-Image Technology \& Internet-Based Systems (SITIS) (pp. 197202). IEEE

6. Truong, X., Mitamura, M., Kono, Y., Raghavan, V., Yonezawa, G., Do, T., Tien Bui, D. and Lee, S., 2018. Enhancing prediction performance of landslide susceptibility model using hybrid machine learning approach of bagging ensemble and logistic model tree. Applied Sciences, 8(7), p.1046.

7. Oh, H.J. and Lee, S., 2017. Shallow landslide susceptibility modeling using the data mining models artificial neural network and boosted tree. Applied Sciences, 7(10), p.1000.

8. Ramesh, M.V., 2009, June. Real-time wireless sensor network for landslide detection. In 2009 Third International Conference on Sensor Technologies and Applications (pp. 405-409). IEEE.

9. Martha, T.R., Kerle, N., van Westen, C.J., Jetten, V. and Kumar, K.V., 2011. Segment optimization and data-driven thresholding for knowledge-based landslide detection by object-based image analysis. IEEE Transactions on Geoscience and Remote Sensing, 49(12), pp.4928-4943.

10. Dahl, G.E., Sainath, T.N. and Hinton, G.E., 2013, May. Improving deep neural networks for LVCSR using rectified linear units and dropout. In 2013 IEEE international conference on acoustics, speech and signal processing (pp. 8609-8613). IEEE.

11. Le, Q.V., Ngiam, J., Coates, A., Lahiri, A., Prochnow, B. and $\mathrm{Ng}$, A.Y., 2011, June. On optimization methods for deep learning. In Proceedings of the 28th International Conference on International Conference on Machine Learning (pp. 265-272). Omnipress. 
12. Liao, X., Xue, Y. and Carin, L., 2005, August. Logistic regression with an auxiliary data source. In Proceedings of the 22nd international conference on Machine learning (pp. 505-512). ACM.

13. Breiman, L., 2001. Random forests. Machine learning, 45(1), pp.5-32.

14. Hu, W., Hu, W. and Maybank, S., 2008. Adaboost-based algorithm for network intrusion detection. IEEE Transactions on Systems, Man, and Cybernetics, Part B (Cybernetics), 38(2), pp.577-583.

15. Zhang, M.L. and Zhou, Z.H., 2007. ML-KNN: A lazy learning approach to multi-label learning. Pattern recognition, 40(7), pp.2038-2048.

16. Mathur, A. and Foody, G.M., 2008. Multiclass and binary SVM classification: Implications for training and classification users. IEEE Geoscience and remote sensing letters, 5(2), pp.241-245.

17. Bradley, A.P., 1997. The use of the area under the ROC curve in the evaluation of machine learning algorithms. Pattern recognition, 30(7), pp.1145-1159. 